\title{
Adult cystic fibrosis: association of acute pulmonary exacerbations and increasing severity of lung disease with auxotrophic mutants of Pseudomonas aeruginosa
}

\author{
Rowena F H Taylor, Margaret E Hodson, Tyrone L Pitt
}

\begin{abstract}
Background-Pseudomonas aeruginosa has been located in the endobronchiolar spaces of patients with cystic fibrosis where nutrients may be limited. In these sites it is thought that adaptation of the pathogen might occur and growth factors, present in relative excess, may thus promote survival of the organism. Auxotrophy of pulmonary isolates of $P$ aeruginosa has previously been shown to be a feature of cystic fibrosis and chronic lung sepsis; auxotrophic isolates have additional nutritional requirements to the prototrophic "wild types" of the species. A study was therefore carried out to determine whether the proportion of auxotrophs differs between stable and acutely ill patients, or correlates with the extent of underlying disease.
\end{abstract}

Methods-Sputum samples were cultured for $P$ aeruginosa and tested for auxotrophy by spreading serial dilutions of homogenised sputum on to a minimal medium which supports only prototrophs, and a complete medium which supports both nutritional types. The proportion of auxotrophs to prototrophs was determined and growth factors of confirmed auxotrophs were identified.

Results-Thirty two (86\%) of 37 adults with cystic fibrosis infected with $P$ aeruginosa harboured auxotrophs; methionine dependent mutants were isolated from seven of 16 patients tested (44\%). More than $50 \%$ of the total number of colonies were auxotrophic in 19 of 26 samples (73\%) from patients with acute exacerbations and in only six of 15 samples (40\%) from clinically stable patients. In four patients from whom samples in both the acute and stable states were available, the proportion of auxotrophs fell in the sample taken when stable. Auxotrophs predominated in all samples from 11 of those patients with very severe underlying lung disease, in contrast to 13 of 30 samples from patients with less severe disease. There was no association between the percentage of auxotrophs and the presence of other respiratory pathogens.

Conclusions-The majority of adults with cystic fibrosis infected with $P$ aeruginosa harbour auxotrophs in the sputum. A significant proportion of acutely ill patients and those with severe underlying disease have a preponderance of auxotrophs in the sputum compared with stable patients and those with less severe disease.

(Thorax 1993;48:1002-1005)

Pulmonary disease in patients with cystic fibrosis is characterised by viscid mucosal secretions which impair natural pulmonary host defence mechanisms ${ }^{1}$ and present a mechanical barrier to endogenous and exogenous antibacterial agents. The range of bacterial species which infect patients with cystic fibrosis is curiously restricted and consists primarily of Pseudomonas aeruginosa, Staphylococcus aureus, Haemophilus influenzae, and Pseudomonas cepacia. These opportunistic pathogens adapt to the abnormal pulmonary environment and the Pseudomonas species, in particular, are seldom eradicated despite appropriate therapy. ${ }^{2}$ In response to infection, inflammatory changes occur which are themselves implicated in further structural damage. ${ }^{3}$ The patient is thereby rendered more susceptible to infection and a cycle of infection and inflammation is established. ${ }^{45}$

$P$ aeruginosa has been found in the endobronchiolar spaces $^{6}$ in which Speert et $a l^{7}$ have proposed that nutrients are limited. We speculated that nutritional adaptation of $P$ aeruginosa might occur in an environment of nutrient imbalance and that growth factors may be present in relative excess within the bronchial mucosa of patients with cystic fibrosis and promote survival of the adaptive organism.

Auxotrophs are bacteria that require growth substances additional to those required by the wild type of the same species (known as prototrophs); in the case of $P$ aeruginosa, prototrophs are supported by minimal media alone. We have shown previously that auxotrophy of $P$ aeruginosa is a frequent characteristic of sputum isolates from patients with cystic fibrosis and non-cystic fibrosis bronchiectasis, that in cystic fibrosis isolates methionine is the single additional factor most often required, ${ }^{8}$ and that, in vitro, auxotrophic isolates are more resistant to antipseudomonal agents than are prototrophs. ${ }^{9}$ We have now examined whether 
the proportion of auxotrophs differs between stable and acutely ill patients and correlates with the extent of underlying lung disease and the presence of other respiratory pathogens. We have also surveyed the auxotrophic requirements of isolates from single sputum samples and from repeated specimens from the same patient.

\section{Methods}

\section{PATIENTS}

Forty one samples were studied from 37 patients (19 men) with cystic fibrosis (mean age 28 , median 26, range 16-67 years). Samples were taken from 22 randomly selected inpatients with acute infective pulmonary exacerbations, 11 stable outpatients, and four patients who were tested both as acutely ill inpatients and stable outpatients. Eleven patients had very severe lung disease (died or received heart-lung transplants within 12 months), 17 had severe lung disease (forced expiratory volume in one second $\left(\mathrm{FEV}_{1}\right)<40 \%$ predicted normal value),$^{10}$ five had moderate disease $\left(\mathrm{FEV}_{1} 40-60 \%\right)$, and four had only mildly impaired lung function $\left(\mathrm{FEV}_{1}>60 \%\right)$. Of the 26 inpatient samples the numbers from patients with very severe, severe, moderate and mild disease were eight, 12, two, and four respectively, and of the 15 outpatient samples the numbers were three, seven, four, and one respectively. Twenty six patients were receiving long term nebulised antibiotics (aminoglycosides, colistin, or both), 17 were receiving inhaled steroids, and all inpatients were receiving intravenous antipseudomonal agents (usually a combination of a penicillin derivative with an aminoglycoside) in conjunction with oxygen therapy, bronchodilators, and physiotherapy. The mean duration of intravenous antibiotic therapy before sampling was nine days (range 2-14, median nine days). All patients were taking pancreatic enzyme supplements and eight were diabetic.

\section{SPUTUM CULTURE}

Manually homogenised sputum was serially diluted $\left(10^{-2}\right.$ to $\left.10^{-8}\right)$ in Ringer's solution and $100 \mu \mathrm{l}$ aliquots of each dilution were spread uniformly on to both a minimal salt medium ${ }^{11}$ which supports only the growth of prototrophs, and King's " $A$ " agar ${ }^{12}$ which supports both nutritional types. After aerobic incubation at $37^{\circ} \mathrm{C}$ for 48 hours the colonies on each plate were counted to determine the total colony count and the proportion of auxotrophs to prototrophs. Isolates which produced the characteristic pigment pyocyanin were accepted to be $P$ aeruginosa and non-pigmented isolates were tested for their ability to oxidise glucose, produce cytochrome oxidase, hydrolyse arginine, and reduce nitrate. ${ }^{13}$

Sputum samples from 24 patients were taken at the same time as the auxotrophy evaluation and cultured for other respiratory pathogens; inpatient samples from a further five patients were processed within 24 hours.
SPECIFIC GROWTH FACTORS

Individual colonies were tested for auxotrophy as previously described ${ }^{8}$ and specific requirements of confirmed auxotrophs were identified by modification ${ }^{8}$ of Holliday's experimental method. ${ }^{14}$

\section{STATISTICAL METHODS}

Comparison of proportions was made by the $\chi^{2}$ test with Yates' correction where appropriate.

\section{Results}

Auxotrophic mutants of $P$ aeruginosa were present in the fresh sputum of 35 of 41 (85\%) sputum samples from 37 patients with cystic fibrosis. In a comparison of stable with acutely ill patients, the majority $(>50 \%)$ of Pseudomonas colonies from each sputum sample were auxotrophic in 19 of 26 inpatients with acute pulmonary exacerbations $(73 \%)$, and in six of 15 stable outpatients $(40 \%)(p<0.05)$

Four patients with cystic fibrosis sampled at random when acutely ill and also when stable harboured a greater proportion of auxotrophs when ill than when stable. The proportion of auxotrophs ranged from $56 \%$ to $98 \%$ in samples taken during acute exacerbations and from $20 \%$ to $52 \%$ in samples taken when stable (fig).

Auxotrophs accounted for more than $50 \%$ of the total Pseudomonas count in all of the 11 patients with very severe underlying lung dysfunction (including three who were tested when stable) and in only 13 of 30 samples from 26 patients with less impairment of lung function $(p<0.01)$. Total colony counts of $P$ aeruginosa ranged from $1 \times 10^{7}$ to $>1 \times$ $10^{9} \mathrm{cfu} / \mathrm{ml}$; there was no relation between the proportion of auxotrophs and the total Pseudomonas colony count or the presence of other sputum pathogens isolated concurrently from 29 of the 37 patients. $S$ aureus was cultured from five patients, Candida albicans $>10^{2} / \mathrm{ml}$ from $16, P$ cepacia from three, and Aspergillus fumigatus from three others.

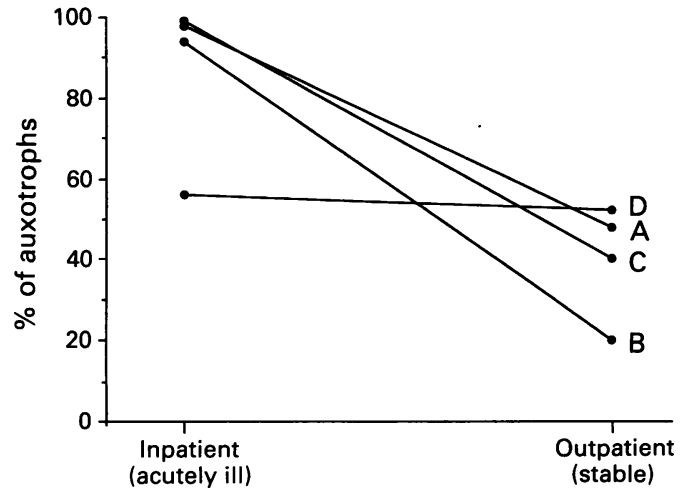

Percentage of auxotrophs in the sputum of four patient with cystic fibrosis sampled when acutely ill and when stable. The time intervals between sampling were 49, 51 69 , and 23 weeks for patients $A, B, C$, and $D$ respectively. All patients were receiving intravenous and nebulised antibiotics when acutely ill and only nebulised agents when stable. 
Table 1 Intrasputum variation of growth requirements of pairs of isolates of $P$ aeruginos a from single sputum samples of 12 patients with cystic fibrosis

\begin{tabular}{|c|c|c|}
\hline \multirow{2}{*}{$\begin{array}{l}\text { Source of } \\
\text { sample }\end{array}$} & \multicolumn{2}{|c|}{ Growth requirements ${ }^{\star}$} \\
\hline & Same/different & Specific \\
\hline Inpatient & Different & $\begin{array}{l}\text { Alternative in } \\
\text { both }\end{array}$ \\
\hline Inpatient & Same & Proline \\
\hline Inpatient & Same & Multiple \\
\hline Outpatient & Same & Multiple \\
\hline Inpatient & Same & Methionine \\
\hline Inpatient & Different & 1 alternative, 1 methionine \\
\hline Inpatient & Same & Methionine \\
\hline Inpatient & Different & $\begin{array}{l}1 \text { not identified, } \\
1 \text { aspartic acid }\end{array}$ \\
\hline Inpatient & Same & Methionine \\
\hline Inpatient & Unknown & Not identified \\
\hline Inpatient & Same & Methionine \\
\hline Inpatient & Same & Methionine \\
\hline
\end{tabular}

${ }^{\star}$ Growth requirements (after Holliday ${ }^{14}$ ): alternativemutant requiring either one or another factor, for example, serine or methionine; single-mutant requiring only one factor; multiple-mutant requiring two or more factors; not identified-requirements absent in factors tested.

\section{SPECIFIC GROWTH FACTORS}

Intrasputum variation

At least two single colonies of auxotrophs, irrespective of colonial morphology, were selected from the primary culture of 12 patients with cystic fibrosis (nine inpatients) for growth factor identification to assess intrasputum variation. Of 12 auxotrophic pairs tested, eight required the same growth factor; as in our previous study methionine was the most frequently required single amino acid (table 1).

\section{Variation with time}

The constancy of auxotrophic $P$ aeruginosa over time was examined in the sputum of nine patients with cystic fibrosis. Table 2 shows that for seven patients there was no variation in growth factor requirements in the two samples taken one or more weeks apart. However, different auxotrophs were identified in the patient from whom samples were taken over the longest time interval (52 weeks).

\section{Methionine dependency}

Overall, methionine dependent strains were present in seven of 16 patients with cystic fibrosis (44\%) and in four of 12 with acute pulmonary exacerbations $(33 \%)$.

Table 2 Constancy of auxotrophic factors of $P$ aeruginosa with time in sputum samples from nine patients with cystic fibrosis

\begin{tabular}{|c|c|c|c|}
\hline \multirow[b]{2}{*}{ Source of paired samples } & \multirow{2}{*}{$\begin{array}{l}\text { Sampling } \\
\text { interval } \\
\text { (weeks) }\end{array}$} & \multicolumn{2}{|c|}{ Growth requirements ${ }^{\star}$} \\
\hline & & Same/different & Specific \\
\hline Both outpatient & 52 & Different & 1 methionine, 1 leucine \\
\hline Both inpatient & 1 & Same & Methionine \\
\hline 1 inpatient, 1 outpatient & 37 & Same & Thiamine \\
\hline 1 inpatient, 1 outpatient & 12 & Same & Alternative \\
\hline 1 inpatient, 1 outpatient & 12 & Same & Methionine \\
\hline Both outpatient & 35 & Same & Methionine \\
\hline Both inpatient & 32 & Same & Methionine \\
\hline Both inpatient & 1 & Unknown & Not identified \\
\hline Both inpatient & 12 & Same & Alternative \\
\hline
\end{tabular}

^For definition of growth requirements see footnote to table 1.

\section{Discussion}

We have found for the first time that most adults with cystic fibrosis infected with $P$ aeruginosa harbour auxotrophic mutants in the sputum (86\%). Furthermore, a significant proportion of acutely ill patients and those with very severe underlying lung disease have a preponderance of auxotrophs in the sputum compared with stable patients and those with less severe disease.

In the light of our findings two further questions arise. Firstly, are auxotrophs of $P$ aeruginosa induced by a relative excess of certain substrates in the lungs of patients with cystic fibrosis and non-cystic fibrosis bronchiectasis, or by antibiotic activity in such patients, or both? Secondly, are the pathogenic effects of auxotrophs greater than those of prototrophs?

At present the mechanisms underlying the selection of auxotrophic mutants of $P$ aeruginosa in cystic fibrosis are poorly understood but the answers to the above questions, together with the knowledge that auxotrophs are more resistant than prototrophs to antipseudomonal agents, might explain how this pathogen persists despite antipseudomonal therapy.

The need for additional growth factors by some members of a bacterial species implies that a specific biosynthetic defect has developed. It follows that auxotrophs can survive in vivo only if the end product of the defective pathway is present in excess within the host environment. Indeed, the relative excess of such factors may, by a negative feedback mechanism, inhibit an enzyme within its own synthetic pathway, and mutants which depend on the substrate in excess may be selected. It is therefore of interest that in pulmonary secretions of patients with suppurative lung disease, including bronchiectasis, there is an excess of glycoprotein ${ }^{15}$ and human DNA. ${ }^{16}$

The growth requirements of isolates from non-cystic fibrosis bronchiectatic patients have not, as yet, been evaluated. In both noncystic fibrosis and cystic fibrosis bronchiectasis, antibiotic treatment may contribute to the selection of auxotrophic $P$ aeruginosa in a similar way to the selection of thymidine dependent strains of $S$ aureus $^{17}$ in patients with cystic fibrosis after long term treatment with cotrimoxazole. It has been postulated that thymidine, a major end product of the folate pathway, is procured by $S$ aureus from degraded DNA, thus overriding the trimethoprim effect and permitting survival of the pathogen. ${ }^{17}$ In order to delineate the effect of disease severity on the induction of auxotrophy and differentiate this from the effect of antibiotic exposure, sequential sputum samples taken before and after antibiotic therapy need to be tested. Auxotrophy of $P$ aeruginosa isolates in non-cystic fibrosis bronchiectasis needs to be evaluated further.

The amino acid composition of sputum from cystic fibrosis and non-cystic fibrosis patients needs to be evaluated in order to account for the preponderance of methionine 
dependent $P$ aeruginosa auxotrophs in sputum from patients with cystic fibrosis. It is of interest that serum levels of the key methionine precursor, sulphadenosylmethionine, are elevated in cystic fibrosis. ${ }^{18}$ Moreover, a report by Tower et al ${ }^{19}$ of in vitro growth suppression of Klebsiella pneumoniae by microbial methionine synthetase inhibitors invites similar experimentation with $P$ aeruginosa. As methionine dependent colonies of $P$ aeruginosa are now shown to be present in one third of acutely ill cystic fibrosis patients and in nearly half ( $44 \%)$ of all patients with cystic fibrosis, the therapeutic possibility of inhibition of microbial methionine synthesis, acting synergistically with conventional antibiotics, should be explored in patients with this disease.

We thank Dr H Gaya, Consultant Microbiologist, the staff of the Microbiology Laboratory at the Royal Brompton Hospital, the Microbiology Laboratory at the Royal Brompton Hospital, and Mrs PS Nicholson from the Division of Hospital Infection (DHI), Central Public Health Laboratory for their help. We are also grateful to Dr BD Cookson, Director of
DHI, Central Public Health Laboratory for his helpful comments, and the Frances and Augustus Newman Research Foundation for financial support (RFHT)

1 Govan JRW, Glass S. The microbiology and therapy of cystic fibrosis lung infections. Rev Med Microbiol 1990; 1:19-28

2 Neijens HJ. Strategies and perspectives in treatment of respiratory infections. Acta Paediatr Scand 1989;363 (Suppl):66-73.

3 Pitt TL. Biology of Pseudomonas aeruginosa in relation to pulmonary infection in cystic fibrosis. $f \mathrm{R} \mathrm{Soc} \mathrm{Med}$ 1986;12(Suppl):13-8.

4 Cole PJ. A new look at the pathogenesis and management of persistent bronchial sepsis; a vicious cycle hypothesis and its logical therapeutic connotations. In: D sepsis. Oxford: The Medicine Publishing Foundation, 1984:1.

5 Elborn JS, Shale DJ. Lung injury in cystic fibrosis. Thorax 1990;45:970-3.

6 Baltimore RS, Christie CDC, Walker Smith GJ. Immunohistopathologic localisation of Pseudomonas aeruginos $a$ in lungs from patients with cystic fibrosis. $\mathrm{Am}$ Rev Respir Dis 1989;140:1650-61.

7 Speert DP, Farmer SW, Campbell ME, Musser JM, Lelander RK, Kuo S. Conversion of Pseudomonas aeruginosa to the phenotype characteristic of strains from patients with cystic fibrosis. $\mathcal{F}$ Clin Microbiol 1990; 28:188-94.

8 Taylor RFH, Hodson ME., Pitt TL. Auxotrophy of Pseudomonas aeruginosa in patients with cystic fibrosis. FEMS Microbiol Lett 1992;92:243-6.

9 Taylor RFH, Warner M, George RC, Hodson ME, Pitt TL. Auxotrophic mutants of $P$ aeruginosa: increased resistance to antipseudomonal antibiotics in cystic fibrosis. Med Microbiol Lett 1993;2:25-32.

10 Cotes JE. Lung function: assessment and application in medicine. Oxford: Blackwell, 1979.

11 Clowes RC, Hayes W. Experiments in microbial genetics. Oxford: Blackwell, 1968:184-5

12 King EO, Ward MK, Raney DE. Two simple media for the demonstration of pyocyanin and fluorescin. $7 \mathrm{Lab}$ Clin Med 1954;44:301-7.

13 King A, Phillips I. The identification of pseudomonads and related bacteria in a clinical laboratory. $\mathfrak{f} \mathrm{Med}$ Microbiol 1978;11:165-76.

14 Holliday R. A new method for the identification of biochemical mutants of microorganisms. Nature 1956;178: 987.

15 Reid LM, Bhaskar KR. Macromolecular and lipid constituents of bronchial epithelial mucus. In: Chandler E, Ratcliffe NA, eds. Mucus and related topics. Cambridge: Company of Biologists, 1989:201-19.

16 Matthews LW, Spector JS, Lemm J, Potter JL. Studies on pulmonary secretions: 1 . The overall chemical composition of pulmonary secretions from patients with cystic tion of pulmonary secretions from patients with cystic
fibrosis, bronchiectasis and laryngectomy. Am Rev Respir Dis 1963;88:199-204.

17 Tablan OC. Prevalence of thymidine-dependent Staphylococcus aureus in patients with cystic fibrosis. F Clin Microbiol 1987;25:1258-61.

18 Schofield D, Bilton D, Rieley F, Webb AK, Bottiglieri T, Braganza JM. Aberrant methionine metabolism in cystic fibrosis. Clin Sci 1992;83(Suppl 27):12P (abstract)

19 Tower PA, Johnson LL, Ferro AJ, Fitchen JH, Riscoe MK. Synergistic activity of 5-trifluoromethylthioribose and inhibitors of methionine synthesis against Klebsiella pneumoniae. Antimicrob Agents Chemother 1991;35: 1557-61. 